\title{
Clinical and microbiological assessment of the results of combined treatment of patients with alveolar osteitis
}

\author{
Gagik Hakobyan', Lazar Yesayan², Davit Mathevosyan³, Gekham Tunyan³ and Laura Gyulamiryan ${ }^{3}$ \\ ${ }^{1}$ Department of Oral and Maxillofacial Surgery Yerevan State Medical University, Armenia \\ ${ }^{2}$ Department of Therapeutic Dentistry, Yerevan State Medical University, Armenia \\ ${ }^{3}$ Department of Oral and Maxillofacial Surgery, Yerevan State Medical University, Armenia
}

\begin{abstract}
Objective: To study the effectiveness of combined treatment in patients with alveolar osteitis after tooth extraction.

Materials and methods: The 87 patients with alveolar osteitis were selected for the study (from 2017 to 2020). Clinical and radiological methods were used to evaluation socket. To conduct a comparative analysis of the treatments results, two groups were formed:

Group I is represented by 44 patients with alveolar osteitis received complex treatment with the inclusion of hyaluronic acid (HA) and magnetic-laser therapy. The II group is represented by 43 patients, with alveolar osteitis received treatment without the inclusion of hyaluronic acid (HA) and magnetic-laser therapy.

To assess the pain syndrome in dynamics, we used visual analogue scale. Patients noted pain intensity in the first visit, as well as in dynamics on the 3rd, 5th, 7th day, respectively.

Group 1 patients, the VAS score indicator shows that by the 5 th procedure in $93 \%$ of cases the pain is completely absent, and only 1 patient has mild pain.

Patients of the 2 group, we observed a similar picture only on the 7-8th day of treatment.

When comparing 2 group the best pain relief was patients first group.

Hyperemia of the mucous membrane of the socket of the extracted tooth on the 5 th day of treatment is completely absent in the 2 nd group only in $32 \%$ of patients of the 1st group, this indicator reaches $98 \%$.

Conclusion: The results of the treatment showed that, complex treatment using Hyaluronic acid (HA) and magnetic-laser therapy reduced the time pain, recovery time, increase the effectiveness of treatment. Thus, on the basis of clinical data, it can be concluded that the anti-inflammatory effect of hyaluronic acid (HA) and magneto-laser therapy is more pronounced than standard treatment methods.
\end{abstract}

\section{Introduction}

From the probable complications post extraction thoot, we can mention alveolar osteitis, bleeding, pain, swelling, trismus, damage to the inferior alveolar nerve, oroantral communication, periodontal problems in surrounding teeth and teeth displacement to noble anatomic spaces. Among the complications associated with the operation of tooth extraction, alveolar osteitis also called dry socket or alveolitis continues to occupy a leading place. Most authors describe following clinical manifestations: spontaneous, constant radiating pain, worse by night. Signs of inflammation around the socket of a previously extracted tooth - such as hyperemia, swelling gums, while the alveolus is empty, or filled with food debris, or necrotic clot $[1,2]$.

Alveolar osteitis is a nest after extraction that shows an open bone that is not covered by a blood clot or healing epithelium and exists within or around the perimeter of the nest or alveoli for several days after the removal procedure [3].

Alveolar osteitis lesions occur in approximately $1 \%$ to $5 \%$ of all extractions and in up to $38 \%$ of mandibular third molar extraction [4].

Food particles and bacterial biofilm may hinder contact of the healing epithelium with the exposed bone, which may prolong the healing time of the alveolar osteitis. This fermentation may result in the formation of toxins or antigens that may irritate the exposed bone, produce an unpleasant taste or halitosis, and cause pain throughout the jaw.

When analyzing the composition of the isolated microorganisms were revealed the following Streptococcus spp isolated in $35.7 \%$, Staphylococcus aureus golden - 12.5\%, Actinomyces spp. $-10.7 \%$, Candida spp. - 8.9\%, Enterococcus spp. - 8.92, Corinobacterium spp. - 7.14\%, Esherichia coli. - 7.14\%, Staphylococcus pneumoniae - 5.35\%, Clostridium spp - 3.6\% [5].

Microorganisms can enter the post-extraction wound from odontogenic and non-odontogenic foci of chronic infection. However, evidence suggests that bacteria is not the main cause of alveolar osteitis dry socket lesions [6].

${ }^{*}$ Correspondence to: Gagik Hakobyan, Department of Oral and Maxillofacial Surgery Yerevan State Medical University, 0028 Kievyan str. 10 ap. 65 Yerevan, Armenia, Tel: (+37410)271146; E-mail: prom_hg@yahoo.com

Received: August 26, 2020; Accepted: October 12, 2020; Published: October 15,2020 
During surgical interventions in the oral cavity, the prevention and development of postoperative edema, hematomas, and local inflammatory reactions are of particular importance. This is due to the anatomical and physiological features of the maxillofacial region: a developed circulatory network, contamination of the oral cavity with microorganisms, the proximity of foci of chronic infection (odontogenic, tonsillogenic, etc.) One of the main reasons for the development of inflammatory complications that occur after tooth extraction is an increase in the virulence of pathogenic microflora with a decrease in the resistance of oral tissues.

Among the causes of alveolar osteitis, the leading role belongs to a pathogenic infection that develops in the absence of a blood clot or its premature destruction in the hole of an extracted tooth. In the literature, there are indications of the existence of factors that can impede the formation or contribute to the destruction of an already formed blood clot [7]. Most authors agree that surgical trauma and the complexity of the operation play a significant role in the development of alveolar osteitis. This may be due to a more active release of direct tissue activators, secondary to bone marrow inflammation, after more complex and, therefore, more traumatic extractions [8].

Some authors believe that the use of vasoconstrictor drugs, administered in conjunction with local anesthetics, leads to prolonged vasospasm and prevents the formation of a blood clot in the tooth socket [9].

There are authors who did not find a significant difference in the distribution of alveolar osteitis of the well after extraction of teeth under infiltration anesthesia compared with conduction [10].

At present, it is accepted that local ischemia due to a vasoconstrictor during local anesthesia does not play a role in the development of alveolar osteitis. They found a significant difference in the distribution of dry socket of the well after extraction of teeth under infiltration anesthesia compared to conduction [11,12].

It is believed that infection of the hole plays a significant role in the development of dry socket, which is facilitated by an unsatisfactory level of oral hygiene. It has been proven that the frequency of dry socket increases in patients with poor oral hygiene.

Risk factors include smoking, surgical trauma, age, gender, medical history, systemic disorder, extraction site, anesthesia experience, operator experience, use of antibiotics before surgery, difficulty of the operation and infection at the previous surgical site, as well as oral contraceptives, menstrual cycle and immediate post-extraction irrigation with saline [13].

Since alveolar osteitis is the most common postoperative complication after removal, many researchers have tried to find a successful method of prevention.

Numerous methods and techniques are offered throughout the existing literature to help prevent alveolar osteitis. However, this area remains a controversial topic, as no method has been universally recognized. A sufficient number of methods have been proposed for the complex treatment of alveolar osteitis, however, none of them is universal, treatment of alveolar osteitis.

Despite the large number of proposed drugs and treatment methods, the problem of prevention of alveolar osteitis remains relevant.

The regular prophylactic use of systemic pre-and/or postoperative antibiotics is challenged due to the development of resistant bacterial strains, possible hypersensitivity and unnecessary destruction of the host commensals.

One of the pharmacological methods used in the prevention of dryness includes the use of antibiotic drugs after extraction and antiseptic rinses. To combat purulent-inflammatory diseases and complications, new types and classes of antibiotics are being developed $[14,15]$.

However, resistance to them is rapidly developing. In this regard, many researchers speak of a serious "crisis of antibacterial therapy". Pathogenic and conditionally pathogenic microflora is in most cases polychemoresistant due to the irrational use of antibiotics.

Several studies have reported that prior to and perioperative use of $0.12 \%$ chlorhexidine reduces the incidence of alveolar osteitis after removal of the third molar of the lower jaw [16]. In addition, various combinations of antibacterial agents in the form of pastes, emulsions, solutions are proposed for the treatment of alveolar osteitis.

Alvogyl (Septodent, Inc., Wilmington, DE) is widely used in dry socket management and is often referred to in the literature. Alvogyl contains butamben (anesthetic), eugenol (analgesic) and iodoform (antimicrobial). Some authors noted a slowdown in healing and inflammation when the nests were filled with alvogil. They did not recommend using it in outlets [17].

When treating alveolar osteitis, the objective is to optimize the lesion such that the socket is optimally capable of forming an enduring layer of epithelium that covers the exposed bone inside the socket. To do this, under anesthesia, can try to cause bleeding into the socket.

Such circumcision can cause the bone to become immediately covered by a blood clot, which will reduce the total number of days that this hypersensitive bone is exposed and helps ensure that the epithelium grows systematically over the remaining exposed bone of the dry socket.

The alveolar osteitis medicament should cover the exposed bone for several days with a resorbable, but durable cover, which will protect the bone from painful mechanical stimulation, food impaction, and bacterial infiltration $[18,19]$.

At the same time, antiseptic medications often cause allergic reactions, and therefore one of the alternatives to medications can be a physical treatment method such as physiotherapy methods.

Physiotherapy methods occupy an important place in rehabilitation complexes in various fields of medicine, being a powerful reserve for increasing the effectiveness of preventive, curative and rehabilitation measures [20].

Physiotherapy methods effectively used as a complement to traditional therapy in dentistry [21]. A known technique for the treatment of alveolar osteitis using ozone therapy [22].

In the last decades, the use of low-level laser therapy has increased as a non-pharmacological adjunct to the treatment with low collateral index in periodontology, implantology, it was pointed that the therapy is known for its efficiency with pain reduction, wound healing and inflammation.

Diode lasers have been shown to have potent bactericidal effects based on this diode laser, carbon dioxide $\left(\mathrm{CO}_{2}\right)$ and Erbium Yttrium, Aluminum, Garnet (Er:YAG) lasers are suitable for irradiating [23-24]. 
Besides the well-established conventional therapies, the lowlevel laser therapy comes as a support, with the goal to help in the reestablishment and faster cure.

In their studies, Kaya $G$, et al. found that treatment of alveolar osteitis was significantly better in patients who were treated with curettage and irrigation, followed by continuous diode laser irradiation, than in patients who were treated with curettage and irrigation, and then applied directly to the socket with Alvogil [25].

The low-level laser therapy is internationally accepted as cell bio-modulator, used to reach ideal therapeutic effects, acting in the reduction of the pain response, with anti-inflammatory effects, stimulating local microcirculation and wounds repair, promoting a rapid recovery, which brings better quality of life to the patient [26].

In a cell level, studies evidence that the laser beam penetrates in the interior of the tissue where it is absorbed by chromophore molecules (light absorbers) that generate the increase adenosine triphosphate quantities (ATP), accelerating the metabolism an producing a physiological response.

The wavelength or laser type ought to be specific for each treatment, although there is not a range of studies with representativeness to define the best type for each dysfunction.

Literature presents the different active means: the red emission laser $(\lambda=630$ to $690 \mathrm{~nm})$ indicated for ulcers, herpes and open wounds healing; the near infrared types (over $700 \mathrm{~nm}$ ), like the gallium arsenide and aluminum diode laser (GaAlAs, $\lambda=790$ to $830 \mathrm{~nm}$ ) which is used for analgesia, swelling, tendinitis, nerve regeneration and chronic ulcers, and the gallium arsenide laser (GaAs, $\lambda=904 \mathrm{~nm}$ ) indicated for the treatment of sports morbidity, postoperative pain and swelling/ edema. Moreover, helium neon gas ( $\mathrm{HeNe}, \lambda=632,8 \mathrm{~nm}$ ) and indium gallium arsenic phosphate (InGaAlP, $\lambda=633 \mathrm{~nm}$ ) are mentioned, both indicated for wounds repai [26]. Known studies on the use of lowintensity laser radiation with a wavelength of $0.63-0.65$ microns and 0.85-0.89 microns accordingly, in the treatment of alveolitis.

The same way that the nonsteroidal anti-inflammatory drugs (NSAIDs), with common use in surgical practice, the low-level laser therapy promotes the same results, however without the side effects. It is worth mentioning that, for immuno-impaired individuals, such as people with diabetes, the benefits described by this therapy are more significant, for they present the same tissue response, also in adverse conditions $[27,28]$.

Magnetic-laser therapy is one of the methods of physiotherapy and accelerates the healing and regeneration of tissues after damage, eliminates pain and swelling. Magnetic-laser field act on the molecules of cell structures, to activate the processes of metabolism of cells and the activity of enzymatic systems, stimulation of nerve cell and lymphocyte respiration. At the tissue level, there is observed: improved microcirculation, accelerated revascularization and reinnervation, decreased intracellular and intercellular edema, increased blood oxygen saturation at the microcirculatory level. The effect of a magnetic field on the surgical field also has an anti-inflammatory, analgesic, regenerating and accelerating tissue healing effect [29,30].

Such a similar therapeutic effect of laser radiation and magnetic field implies an increase in efficiency when used together.

Therefore, the effectiveness of magnetic-laser therapy in the treatment of alveolar osteitis needs to be studied.
Hyaluronic acid (HA) is a glycosaminoglycan is one of the main linear extracellular matrix polysaccharides that can be found in various tissues of the body, especially in connective tissue and synovial fluid. It has many functions, such as the elastic viscosity of synovial fluid in the joints, control of tissue hydration and the mechanism of cell detachment with anti-inflammatory \& anti edematous properties. With the advances in understanding of inflammatory mechanisms and wound healing process associated with thoot socket, numerous extracellular matrix components like hyaluronic acid, chondroitin sulphate and fibronectin are identified as promoters of periodontal healing and regeneration.

In addition, Hyaluronic acid can be used safely in medicine as it is non-immunogenic and non-toxic [31-33]. Hyaluronic acid plays a multifunctional role in the wound healing process. In dentistry, it was first used in the treatment of periodontal diseases such as gingivitis. Wondering to see if there is any there is any beneficial value of local administration of $0.8 \%$ Hyaluronic acid gel formulation in the treatment of dry socket.

\section{Objective}

To study the effectiveness of combined treatment in patients with alveolar osteitis after tooth extraction.

\section{Materials and methods}

The 87 patients with alveolar osteitis were selected for the study (from 2016 to 2020). The ages of the patients ranged between 24 and 53 years (46 males and 41 females). All patients presented alveolar osteitis complications associated with the operation of tooth extraction. Clinical and radiological methods were used to evaluation socket.

The clinical study took into account: the localization of the defect, the presence of an inflammatory process, the volume of the bone and soft tissues of the jaw in the surgical area extracted thoot.

Among patients, most often developed in areas of premolars and molars of the lower jaw (54.9\%), premolars and molars of the upper jaw (19.3\%), front teeth of the upper jaw (16.6\% about). Least of all, this process developed in patients after the removal of the front teeth of the lower jaw (9.2\%). Clinically, alveolar osteitis outlet is revealed in patients after extraction, which shows an open bone not covered by a blood clot.

All patients underwent local and general treatment, including antiseptic treatment, the appointment of anti-inflammatory and antimicrobial agents.

Curettage of dry socket was performed after anesthesia and socket well was washed with a chlorhexidine solution and after that they achieved that the socket was filled with blood and a blood clot formed.

To conduct a comparative analysis of the treatments results, two groups were formed:

Group I is represented by 43 patients whith dry socket received complex treatment with the inclusion of magnetIc-laser therapy, hyaluronic acid (Gengigel ${ }^{\circledR}$ ) was applied to the socket. Patients received magnetic- laser irradiation 7 days with a wavelength of $810 \mathrm{~nm}$ and a density of $100 \mathrm{~mW}$ during $5 \mathrm{~min}$. The exposure was carried out using the apparatus "Milta"(RF,Moscow).

The II group is represented by 45 patients, whith dry socket was observed, received treatment without the inclusion of hyaluronic acid $\left(\right.$ Gengigel ${ }^{\circledR}$ ) and magnetic-laser therapy, nothing was applied to the socket. 
Sample for microbiological examination was taken before starting therapy. Sterile curette was moved to the bottom of respective socket and put into a vial containing anaerobic culture media. Bacteria were identified based on their morphotype i.e. shape, size and motility and were divided into three groups: Gram positive cocci, Gram negative bacilli (straight rods, curved rods, motile rods) and filamentous bacteria. From 100-150 bacteria examined, data was recorded as relative percentage of each of these bacteria. Microbiological sampling at the reexamination was before clinical examination at 1 weeks.

To assess the pain syndrome in dynamics, we used Visual Analogue Scale (VAS) [34]. Visual analogue scale (VAS) is a straight line, the starting point of which corresponds to the absence of pain, and final - intolerable pain. At equal intervals under the line are numbers from 1 to 10 , indicating the intensity pain and characterized by supporting words: absent, weak, moderate, strong, unbearable.

Patients noted pain intensity in the first visit, as well as in dynamics on the 3rd, 5th, 7th day, respectively. Patients were instructed that a score of 1 to 3 was indicative of mild pain, 4 to 6 was indicative of moderate pain, and 7 to 10 was indicative of sever. Patients were instructed to note the intensity of pain at the first visit, as well as in dynamics on the 3 rd, 5th, 7th day, respectively.

The study was reviewed and approved by the Ethics Committee of the of the Yerevan State Medical University after M. Heratsi (protocol N12, 05.9.17) and in accordance with those of the World Medical Association and the Helsinki Declaration.

\section{Results}

It was found that in patients undergoing of hyaluronic acid (Gengigel -) and magnetic-laser therapy, complete replacement of blood clot with granulation tissue and the process of marginal epithelization was noted by the 7 th procedure.

Group 1 patients, the VAS score indicator shows that by the 5th procedure in $93 \%$ of cases the pain is completely absent, and only 1 patient has mild pain.

Patients of the 2 group, we observed a similar picture only on the $7-8$ th day of treatment. When comparing 2 group the best pain relief was patients first group.

Hyperemia of the mucous membrane of the hole of the extracted tooth on the 5 th day of treatment is completely absent in the 2 nd group only in $32 \%$ of patients of the 1 st group, this indicator reaches $98 \%$.

In present study, microbiological examinations bacteria were identified based on their morphotype i.e. shape, size and motility and were divided into three groups: Gram positive cocci, Gram negative bacilli (straight rods, curved rods, motile rods) and filamentous bacteria. From 100-150 bacteria examined, data was recorded as relative percentage of each of these bacteria.

There was reduction in the number of gram negative bacilli and relative increase in the number of positive coccoid cells, in all 2 treatment modalities compared to baseline.1 group presented decrease in gram negative bacilli ( $45.2 \%$ at baseline to $27.4 \%$ at 1 weeks) and relative increase in gram positive coccoid cells $(54.80 \%$ at baseline to $72 \%$ at 1 weeks). In group comparison there was no statistically significant difference found in microbiological parameters $(\mathrm{p}>0.05)$. 2 group showed decrease in gram negative bacilli ( $45.2 \%$ at baseline to $30 \%$ at 1 week) and relative increase in gram positive coccoid cells ( $54.80 \%$ at baseline to $70 \%$ at 1 weeks).
Thus, on the basis of clinical data, it can be concluded that the antiinflammatory effect of magneto-laser therapy is more pronounced than standard treatment methods.

\section{Statistical analyses}

Statistical analyses were performed using SPSS software ver. 22.0 (IBM, Armonk, NY, USA), and MedCalc program for Windows. To test the significance of variations in the BOP, PPD, MBL, the t-test was used. The minimum level of statistical significance was set at a value of less than 0.05 .

\section{Discussion}

Alveolar osteitis is a widespread disease, which, according to various authors, accounts for $24-35 \%$ of all cases of complications encountered in patients after tooth extraction $[35,36]$.

One of the main reasons for the development of inflammatory complications that occur after tooth extraction is an increase in the virulence of pathogenic microflora against the background of a decrease in the resistance of tissues of the oral cavity. Therefore, one of the most important issues of modern dentistry is the treatment of the wound surface after surgery.

Among the causes of alveolar osteitis, the leading role belongs to a pathogenic infection, which develops in the absence of a blood clot or its premature destruction in the hole of the extracted tooth. Most often, the cause of the development of alveolar osteitis is Staphylococcus aureus and epidermal, diplococcus, streptococcus [37-39].

It is well known that postoperative inflammatory reactions reach a maximum level 2 days after surgery and usually resolve within 1 week. Thus, the first week after surgery has a strong impact on the quality of life of patients, and it is extremely important to eliminate the associated factors that affect the initial phases of wound healing. Therefore, one of the most important issues in modern dentistry is the issue of treatment of alveolar osteitis. Despite years of research, little progress has been made in treating this common and unpleasant postoperative condition in patients.

All this makes us look for new approaches to the prevention and treatment of purulent-inflammatory complications. It should be noted that the search for new methods for preventing the development of complications after tooth extraction surgery and their treatment continues to this day. Of great importance in solving this problem is local treatment, which is carried out using various wound dressings. Modern coatings should prevent the development of infection and its penetration into the wound, protect the wound from injury, absorb the resulting exudate, and prevent its accumulation under the dressing.

At the same time, there are sufficient grounds for the development and inclusion in the program of combating postoperative complications of physical factors. Possessing anti-inflammatory, antibacterial, analgesic, stress-limiting. immunomodulatory and regenerative action.

This diversity reflects the relevance of the problem itself and requires further study.

One of these physiotherapeutic factors is the low-intensity lasers and electromagnetic field, the use of which using modern electronic technologies, make it possible to choose the necessary optimal frequency for a particular patient, due to which all physiological effects are significantly enhanced and the effectiveness of this unique physiotherapeutic factor is $[40,41]$. 
Diode lasers have been shown to photobiomodulatory effects promoting wound healing and tissue regeneration. Diode lasers stimulate fibroblasts and osteoblasts, causeincr eased production of RNA messengers, which leads to significant collagen production during tissue healing [42-45].

Magnetic-laser supportive therapy may be considered an adjunct to the conventional surgical treatments of alveolar osteitis [46-47].

It is advisable to include a course of magnetic-laser therapy in the complex of therapeutic measures for dry sockets that occurs after removal.

The use of magnetic-laser therapy reduces the inflammatory manifestations of dry sockets, which developed after tooth extraction, and accelerates the reparative processes in the mucous membrane of the hole of the extracted tooth. One of the reasons for such rapid changes should be the normalization of microcirculation in the area of inflammation due to the reduction of thrombosis in microvessels and the activation of anti-inflammatory systems under the influence of oxygen ions. Effectiveness of magnetic-laser therapy on pain reduction can be explained by the fact laser light absorbed by nociceptors, exert an inhibitory effect on A and C pain fibers, which slows conduction velocity, and suppresses neurogenic inflammation [48].

Based on this, research is needed to illustrate the clinical benefits of treatment alveolar osteitis using magnetic-laser therapy.

The developed optimal parameters of magnetic-laser therapy (exposure time of 5 minutes in intensive mode for 5-7 days after tooth extraction helps to normalize the course of the wound process of the socket of a extracted tooth. To achieve an optimal clinical result, at least 7 sessions of magnetic-laser therapy are necessary.

The results of our research have shown that Magnetic-laser therapy have shown promising therapeutic effect in treatment of alveolar osteitis. Magnetic-laser therapy (exposure time of 5 minutes in intensive mode for 5-7 days after tooth extraction helps to normalize the course of the wound process of the socket of a extracted tooth. To achieve an optimal clinical result, at least 7 sessions of magnetic-laser therapy are necessary.

The Visual Analogue Scale (VAS) pain scores for each group patients with alveolar osteitis at each time were analyzed. A reduction in the duration of pain in patients of the first group compared to patients second group shows the effectiveness of magnetic-laser therapy. Magnetic-laser irradiation will significantly decrease pain, swelling, inflammation and stimulate the covering of the exposed bone through the stimulation of fibroblasts. Magnetic-laser therapy promote to increase blood flow by vasodilation and also reduces edema.

Hyaluronic acid (HA) plays a multifaceted role in regulating various biological processes and maintaining homeostasis in the body. HA is an essential component of connective tissue, and plays an important role in maintaining the elasticity and viscosity of the extracellular matrix [49-51].

Several studies in the literature report that HA reduces symptoms, especially pain in patients with osteoarthritis. Gotoh et al reported that HA has an analgesic effect by covering the bradykinin receptors in synovial tissues and supporting an analgesic role [52]. In addition, they suggested a correlation between the molecular weight of hyaluronic acid (HA) and its analgesic effect. Nelson et al investigated the efficacy of oral administration of hyaluronic acid (HA) using spectral analysis of serum and joint fluid in patients with osteoarthritis of the knee joint [53].
Gokmen, et al. stated that the hyaluronic acid (HA) applied after the third removal of the molars showed less leukocyte infiltration and greater angiogenesis [54]. Coray, et al. evaluated the efficacy of hyaluronic acid (HA) spray after the third molar removal and indicated that post-hyaluronic acid (HA) surgery reduces swelling and may lead to a decrease in alveolar osteitis [55]. Juhas et al. reported a decrease in the size of the wound, although there was a decrease in pain [56]. Similarly, Onisti, et al. in addition to reducing the size of the wound, they also reported a decrease in pain in half of the patients and without pain in most others [57]. Ballini, et al. studied the effect of esterified hyaluronic acid in the treatment of infrabony periodontal defects $[58,59]$.

Based on above concept a treatment modalities, hyaluronic acid (HA) gel was used for the adjunctive in present study. In our study, a decrease in pain in the hyaluronic acid (HA) group may be due to the anti-inflammatory contribution of hyaluronic acid (HA).

Thus, it was concluded that hyaluronic acid (HA) reduces not only pain, but also local and systemic inflammation. In our research the use of combined exposure of a constant magnetic field and laser radiation was found to promote treatments effectivens alveolar osteitis.

\section{Conclusion}

The results of the treatment showed that, complex treatment using hyaluronic acid (HA) and magnetic-laser therapy reduced the time pain, recovery time, increase the effectiveness of treatment. Thus, on the basis of clinical data, it can be concluded that the anti-inflammatory effect of hyaluronic acid (HA) and magneto-laser therapy is more pronounced than standard treatment methods.

\section{Conflict of interest}

None.

\section{Funding}

None.

\section{References}

1. John Mamoun (2018) Dry Socket Etiology, Diagnosis, and Clinical Treatment Techniques. Assoc Oral Maxillofac Surg 44: 52-58. [Crossref]

2. Blum IR (2002) Contemporary views on dry socket (alveolar osteitis): a clinical appraisal of standardization, aetiopathogenesis and management: a critical review. Int J Oral Maxillofac Surg 31: 309-317. [Crossref]

3. Nitzan DW (1983) On the genesis of "dry socket”. J Oral Maxillofac Surg 41: 706-710 [Crossref]

4. Bowe DC, Rogers S, Stassen LF (2012) The management of dry socket/alveolar osteitis. J Ir Dent Assoc 57: 305-310. [Crossref]

5. Tarakji B, Saleh A, Umair A (2015) Systemic review of dry socket: aetiology, treatment and prevention. J Clin Diagn Res 9: ZE10-ZE13. [Crossref]

6. Colby RC (1997) The general practitioner's perspective of the etiology, prevention, and treatment of dry socket. Gen Dent 45: 461-467. [Crossref]

7. Hedstrom L (2007) Effect estimates and methodological quality of randomized controlled trials about prevention of alveolar osteitis following tooth extraction: a systematic review. Oral Surg Oral Med Oral Pathol Oral Radiol Endodont 103: 8-15 [Crossref]

8. Nusair YM, Abu Younis MH (2007) Prevalence, clinical picture, and risk factors of dry socket in a Jordanian Dental Teaching Center. J Contemp Dent Pract 8: 53-63. [Crossref]

9. Lehner T (1958) Analysis of one hundred cases of dry socket. Dental Practitioner and Dental Record 8: 275-279.

10. Ishihama K, Kimura T, Yasui Y, Komaki M, Ota Y (2006) Azithromycin as prophylaxis for the prevention of postoperative infection in impacted mandibular third-molar surgery. J Infect Chemother 12: 31-35. [Crossref] 
11. Wiśniewska I, Slósarczyk A, Myśliwiec L, Sporniak-Tutak K (2009) Lincomycin applied to the alveolus on TCP carrier and its effect on wound healing after surgical extraction of a third molar. Ann Acad Med Stetin 55: 59-64. [Crossref]

12. Larsen PE (1991) The effect of a chlorhexidine rinse on the incidence of alveolar osteitis following the surgical removal of impacted mandibular third molars. J Oral Maxillofac Surg 49: 932-937. [Crossref]

13. Nusair YM, Abu Younis (2007) Prevalence, clinical picture, and risk factors of dry socket in a Jordanian Dental Teaching Center. J Contemp Dent Pract 8: 53-63. [Crossref]

14. Muhammad AS (2010) Pathogenesis and management of dry socket (alveolar osteitis). Pak Oral Dent J 38: 323-326.

15. Penarrocha-Diago M, Sanchis J.M., S'aez U. et al. Oral hygiene and postoperative pain after mandibular third molar surgery. Oral Surg Oral Med Oral Pathol Oral Radiol Endodont 92: 260-264

16. Syrjänen SM, Syrjänen KJ (1979) Influence of Alvogyl on the healing of extraction wound in man. Int J Oral Surg 8: 22-30. [Crossref]

17. Taberner-Vallverdú M, Nazir M, Sánchez-Garcés MÁ, Gay-Escoda C (2015) Efficacy of different methods used for dry socket management: a systematic review. Med Oral Patol Oral Cir Bucal 20: 633-639. [Crossref]

18. Kaya GŞ, Yapici G, Savaş Z, Güngörmüş M (2011) Comparison of alvogyl, SaliCep patch, and low-level laser therapy in the management of alveolar osteitis. J Oral Maxillofac Surg 69: 1571-1577. [Crossref]

19. Sperandio FF, Giudice FS, Corrêa L, Décio Júnior SP, Hamblin MR, et al. (2013) Low-level laser therapy can produce increased aggressiveness of dysplastic and oral cancer cell lines by modulation of Akt/mTOR signaling path way. J Biophotonics 6: 839-847. [Crossref]

20. Fekrazad R, Chiniforush N, Bouraima SA, Aslani MVM, Zare M, et al. (2012) Low level laser therapy in management of complications after intra oral surgeries. $J$ Lasers Med Sci 3: 135-140.

21. Karu T (1999) Primary and secondary mechanisms of action of visible to near-IR radiation on cells. J Photochem Photobiol B 49: 1-17 [Crossref]

22. Géza Bálint, Péter Bálint (2013) The use of physiotherapy tools in medical treatment The use of heat, light, electric, magnetic, ultrasound and shock wave therapy as well as medical massage in Hungarian medical practice. Orv Hetil 154: 1905-1911. [Crossref]

23. Gadottiet IC (2018) Dentists' Awareness of Physical Therapy in the Treatment of Temporomandibular Disorders: A Preliminary Study. Hindawi Pain Research and Management.

24. Guerra OC (1997) Aplicaion de la ozonoterapa en al tratamiento de la alveolitis. Revista Cubana de Estomatolgia 34: 21-24

25. Guo-Hao Lin, Fernando Suárez López del Amo, Hom-Lay Wang (2018) Laser therapy for treatment of peri-implant mucositis and peri-implantitis: An American Academy of Periodontology best evidence review. J Periodontol 89: 766-782 [Crossref]

26. Behdin S, Monje A, Lin GH, Edwards B, Othman A, et al. (2015) Effectiveness of laser application for periodontal surgical therapy: systematic review and meta-analysis. $J$ Periodontol 86: 1352-1363 [Crossref]

27. Aupois R (2006) Postextraction alveolitis. Inf Dent 88: 29-32.

28. MILETO TN, AZAMBUJA FG (2017) Low-intensity laser efficacy in postoperative extraction of third molars. RGO Rev Gaúch Odontol 65: 1.

29. Freitas LF, Hamblin MR (2016) Proposed Mechanisms of Photobiomodulation or Low-Level Light Therapy. IEEE J Sel Top Quantum Electron 22: 7000417. [Crossref]

30. Maria Emília de Abreu Chaves (2014) Effects of low-power light therapy on wound healing: LASER x LED*. An Bras Dermatol 89: 616-623. [Crossref]

31. Marycz K, Kornicka K, Röcken M (2018) Static magnetic field (SMF) as a regulator of stem cell fate-new perspectives in regenerative medicine arising from an underestimated tool. Stem Cell Rev 14: 785-792. [Crossref]

32. Hakobyan Gagik, Lazar Yesayan, Arman Seyranyan, Davit Matevosyan (2020) The use of magnetic-laser therapy of patients with post-dental implant neuropathy. Journal of Novel Physiotherapy and Physical Rehabilitation 7: 009-012.

33. Hamida Refai Dalia Radwan, Nermeen Hassanien (2014) Radiodensitometric Assessment of the Effect of Pulsed Electromagnetic Field Stimulation Versus Low Intensity Laser Irradiation on Mandibular Fracture Repair: A Preliminary Clinical trial. J Maxillofac Oral Surg 13: 451-457. [Crossref]
34. Radice M, Brun P, Cortivo R (2000) Hyaluronan-based biopolymers as delivery vehicles for bone-marrow-derived mesenchymal progenitors. J Biomed Mater Res 50: 101-109. [Crossref]

35. Sahayata VN, Bhavsar NV, Brahmbhatt NA (2014) An evaluation of $0.2 \%$ hyaluronic acid gel (Gengigel $(\mathrm{R})$ in the treatment of gingivitis: A clinical and microbiological study. Oral Health Dent Manag 13: 779-785. [Crossref]

36. Couper M, Tourangeau R, Conrad F (2006) Evaluating the effectiveness of visual analog scales: A web experiment. Soc Sci Comput Rev 24: 227-245.

37. Abu Younis MH, Abu Hantash RO (2011) Dry socket: frequency, clinical picture, and risk factors in a palestinian dental teaching center. Open Dent J 5: 7-12. [Crossref]

38. Kolokythas Antonia, Olech Eliza, Miloro Michael (2010) Alveolar osteitis: comprehensive review \& controversies. Int J Dent 2010: 249073. [Crossref]

39. Haraji A, Motamedi MH, Rezvani F (2010) Can flap design influence the incidence of alveolar osteitis following removal of impacted mandibular third molars? Gen Dent 58: 187-189. [Crossref]

40. Bortoluzzi MC, Manfro R, De Déa BE, Dutra TC (2010) Incidence of dry socket, alveolar infection, and postoperative pain following the extraction of erupted teeth. $\mathrm{J}$ Contemp Dent Pract. 2010;11:E033-40. [Crossref]

41. Mohammed H, Abu Younis, Ra'ed O Abu Hantash (2011) Dry socket: frequency, clinical picture, and risk factors in a palestinian dental teaching center. Open Dent $\mathrm{J}$ 5: 7-12. [Crossref]

42. López-Ramírez M, Vílchez-Pérez MA, Gargallo-Albiol J, Arnabat-Domínguez J, GayEscoda C (2012) Efficacy of low-level laser therapy in the management of pain, facia swelling, and postoperative trismus after third molar extraction to lower. A preliminary study. Lasers Med Sci 27: 559-566. [Crossref]

43. Brignardello-Petersen R, Carrasco-Labra A, Araya I, Yanine N, Beyene J, et al. (2012) Is adjuvant laser therapy is effective for preventing pain, swelling, and trismus after surgical removal of impacted mandibular third molars? A systematic review and metaanalysis. J Oral Maxillofac Surg 70: 1789-801. [Crossref]

44. Sierra SO, Deana AM, Bussadori SK, Mota ACC, Motta LJ, et al. (2015) Effect of low-intensity laser treatment on pain after extraction of impacted mandibular third molars: A randomised, controlled, clinical trial. Br J Oral Maxillofac Surg 53: 9961000. [Crossref]

45. Batinjan G, Zore IF, Rupic I, Juric IB, Zore Z, et al. (2013) Assessing health-related quality of life with antimicrobial photodynamic therapy (APDT) and low level laser therapy (LLLT) after third molar removal. J Lasers Med Sci 4: 120-126. [Crossref]

46. Vartika Kathuria, Jatinder Kaur Dhillon, Gauri Kalra (2015) Low Level Laser Therapy: A Panacea for oral maladies.Laser Ther 24: 215-223. [Crossref]

47. Markovic AB, Todorovic L (2006) Postoperative analgesia after lower third molar surgery: contribution of the use of long-acting local anesthetics, low-power laser, and diclofenac. Oral Surg Oral Med Oral Pathol Oral Radiol Endod 102: 4-8. [Crossref]

48. Darendeliler MA, Darendeliler A, Sinclair PM (1997) Effects of static magnetic and pulsed electromagnetic fields on bone healing. Int J Adult Orthodon Orthognath Surg 12: 43-53 [Crossref]

49. Abdelrahim A, Hassanein HR, Dahaba M (2011) Effect Of pulsed electromagnetic field on healing of mandibular fracture: a preliminary clinical study. J Oral Maxillofac Surg 69: 1708- 1717. [Crossref]

50. Vartika Kathuria, Jatinder Kaur Dhillon, Gauri Kalra (2015) Low Level Laser Therapy: A Panacea for oral maladies. Laser Ther 24: 215-223. [Crossref]

51. Koshal A, Patel P, Robert B, Galgut Peter N (2007) A comparison in postoperative healing of sites receiving non-surgical debridement augmented with and without single application of hyaluronan $0.8 \%$ gel. Oral Health \& Preventive Dentistry 2: 34-38.

52. Xu Y, Höfling K, Fimmers R, Frentzen M, Jervøe-Storm PM (2004) Clinical and microbiological effects of topical subgingival application of hyaluronic acid gel adjunctive to scaling and root planing in the treatment of chronic periodontitis. $J$ Periodontol 75: 1114-1118. [Crossref]

53. Gotoh S, Onaya J, Abe M (1993) Effects of the molecular weight of hyaluronic acid and its action mechanisms on experimental joint pain in rats. Ann Rheum Dis 52: 817 822 [Crossref]

54. Nelson FR, Zvirbulis RA, Zonca B (2015) The effects of an oral preparation containing hyaluronic acid (Oralvisc(R)) on obese knee osteoarthritis patients determined by pain, function, bradykinin, leptin, inflammatory cytokines, and heavy water analyses. Rheumatol Int 35: 43 [Crossref]

55. Gocmen G, Gonul O, Oktay NS (2015) The antioxidant and anti-inflammatory efficiency of hyaluronic acid after third molar extraction. $J$ Craniomaxillofac Surg 43 1033-1037. [Crossref] 
Hakobyan G (2020) Clinical and microbiological assessment of the results of combined treatment of patients with alveolar osteitis

56. Koray M, Ofluoglu D, Onal EA (2014) Efficacy of hyaluronic acid spray on swelling, pain, and trismus after surgical extraction of impacted mandibular third molars. Int $J$ Oral Maxillofac Surg 43: 1399-1403. [Crossref]

57. Juhasz I, Zoltan P, Erdei I (2012) Treatment of partial thickness burns with Znhyaluronan: Lessons of a clinical pilot study. Ann Burns Fire Disasters 25: 82-5 57. [Crossref]
58. Onesti MG, Fino P, Ponzo I (2014) Non-surgical treatment of deep wounds triggered by harmful physical and chemical agents: A successful combined use of collagenase and hyaluronic acid. Int Wound J 13: 22-26. [Crossref]

59. Ballini A, Cantore S, Capodiferro S, Grassi FR (2009) Esterified hyaluronic acid and autologous bone in the surgical correction of the infra-bone defects. Int J Med Sci 6: 65-71. [Crossref]

Copyright: (C2020 Hakobyan G. This is an open-access article distributed under the terms of the Creative Commons Attribution License, which permits unrestricted use, distribution, and reproduction in any medium, provided the original author and source are credited. 